(2) Open Access Full Text Article

REVIEW

\title{
Spotlight on ixekizumab for the treatment of moderate-to-severe plaque psoriasis: design, development, and use in therapy
}

This article was published in the following Dove Press journal:

Drug Design, Development and Therapy

2 June 2017

Number of times this article has been viewed

\author{
Alessandro Giuntal,* \\ Alessandra Ventural,* \\ Maria Sole Chimenti ${ }^{2}$ \\ Luca Bianchi' \\ Maria Esposito' \\ 'Department of Dermatology, \\ ${ }^{2}$ Department of Rheumatology, \\ University of Rome Tor Vergata, \\ Rome, Italy \\ *These authors contributed equally \\ to this work
}

\begin{abstract}
Psoriasis is a chronic inflammatory disease affecting up to $3 \%$ of the general population, associated with discomfort and impaired quality of life. In recent years, the pathogenic cytokine network of psoriasis has been extensively studied leading to the development of new treatments that provide greater efficacy. Interleukin 17A (IL-17A) has been recognized as a crucial cytokine that mediates immunopathogenesis of psoriasis. Ixekizumab-indicated for the treatment of adults with moderate-to-severe plaque psoriasis - is a subcutaneously administered humanized monoclonal antibody that targets IL-17A. A large percentage of patients affected by psoriasis achieved consistent benefits in terms of disease control and rapid onset of action during clinical trials. Overall, ixekizumab brought clinical improvement and a favorable safety profile in phase III trials. Ixekizumab is characterized by consistent efficacy and rapid onset of response; it is not influenced by previous exposure to biologics and has shown good results in areas that are difficult to treat and in severe clinical variants of psoriasis. Ixekizumab has shown significant improvements in the activity of the disease and in those physical functions that inhibit radiographic progression in patients with concomitant involvement of joints. Our data support ixekizumab as a successful therapeutic option for patients affected by moderateto-severe plaque-type psoriasis.
\end{abstract}

Keywords: biologic therapies, IL-17, ixekizumab, psoriasis

\section{Introduction}

Psoriasis is a chronic inflammatory disease affecting up to $3 \%$ of the general population. It is clinically characterized by epidermal hyperproliferation leading to erythematoussquamous skin plaques. Up to $30 \%$ of psoriatic patients may also suffer from seronegative spondyloarthritis. ${ }^{1}$

Psoriasis is nowadays considered a systemic disorder that needs a multidisciplinary approach and an appropriated treatment taking into account different comorbidities. Indeed, several studies demonstrated the association between psoriasis and a number of disease-related comorbidities including blood hypertension and cardiovascular diseases, obesity, type II diabetes, dyslipidemia - singularly or combined in order to configure the metabolic syndrome - nonalcoholic fatty liver disease, anxiety, depression, and inflammatory bowel disease. ${ }^{2-6}$

Psoriasis has been reported to have a notable impact on social relationships, mental health, and work-related activities. ${ }^{6,7}$ Although new molecules have been discovered in the last 2 decades leading to an improvement in the quality of life of patients as well as in skin and joint symptoms, the disease is still not controllable in many patients, in particular in case of long-term patients. ${ }^{8}$
Correspondence: Maria Esposito Department of Dermatology, University of Rome Tor Vergata, Viale Oxford 8I-00I33, Rome, Italy

Tel +390620902743

Fax +3906 20902742

Email espositomaria@virgilio.it
Drug Design, Development and Therapy 20|7:| | |643-|65|

1643

Dovepress f 10

http:!/dx.doi.org/10.2147/DDDTS92128

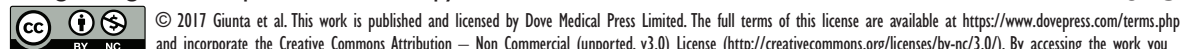

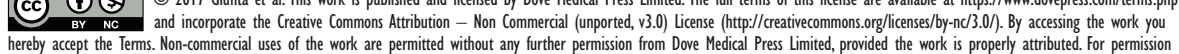
hereby accept the Terms. Non-commercial uses of the work are permitted without any further permisision from Dove Medic
for commercial use of this work, please see paragraphs 4.2 and 5 of our Terms (https.//www.dovepress.com/terms.php). 
Recent discoveries regarding the pathogenesis of the disease have enabled the identification of some new promising targets for psoriasis treatment, particularly interleukin 17 (IL-17). ${ }^{9,10}$

This review will focus on ixekizumab, an anti-IL-17 human monoclonal antibody.

\section{Role of IL-I 7 in the pathogenesis of plaque-type psoriasis}

IL-17 is a family of proinflammatory cytokines first described by Yao et al and comprising IL-17A, IL-17B, IL-17C, IL-17D, IL-17E, and IL-17F secreted by T cells, natural killer cells, mast cells, and neutrophils. ${ }^{11,12}$ IL-17A represents the most investigated cytokine of this family. It is involved in host defense against infections and is implicated in various inflammatory disorders including autoimmune diseases, metabolic disorders, and cancer. IL-17A promotes many events that lead to inflammation, neutrophil recruitment, and host defense throughout the secretions of a multiplicity of molecules as cytokines, chemokines, acute-phase proteins, antimicrobial peptides, mucins, and matrix metalloproteinases. ${ }^{12,13}$

IL-17A is produced predominantly by Th (T helper) 17 cells, a subset of $\mathrm{CD}^{+} \mathrm{T}$ cells. An aberrant production of IL-17A is strongly related to the pathogenesis of psoriasis and other autoimmune diseases such as rheumatoid arthritis, chronic noninfectious uveitis, and Crohn's disease. The block of IL-17A represents a potential target in the treatment of a number of autoinflammatory disorders. ${ }^{13}$ Furthermore, the role of IL-17 in the pathogenesis of psoriatic arthritis (PsA) was assumed when increased levels of IL-17 and tumor necrosis factor alpha (TNF- $\alpha$ ) mRNA expression were observed in the synovial liquid of patients affected by rheumatoid arthritis. ${ }^{14}$ Thereafter, several studies described the relation between IL-1-17, TNF- $\alpha$, IL-6, IL-1, and IL-8 demonstrating that IL-17 enhances TNF- $\alpha$-induced synthesis of interleukins 1, 6, and 8 in skin and synovial fibroblasts acting as a "fine-tuning cytokine" in a functional cooperation mediated by CCAAT/enhancer binding protein family members. ${ }^{15-19}$ Finally, it has been demonstrated that IL-17 induces cartilage collagen breakdown and is involved in osteoclastogenesis and bone resorption through the activation of nuclear factor kappa B ligand. ${ }^{20-22}$

Plaque-type psoriasis has been historically considered as a Th1-mediated disease despite increasing evidence suggesting the significance of the IL-23/T17 axis as a key pathogenic pathway. ${ }^{23-25}$ In particular, elevated levels of IL-17A, IL-17F, IL-22, and IL-23 were detected in human lesional skin, ${ }^{26-29}$ while circulating levels of T17 signature cytokines were higher in psoriatic patients compared to healthy subjects and correlated to the severity of disease..$^{30,31}$

In brief, the main steps involving IL-17 in the pathogenesis of psoriasis are 1) naïve T cell differentiating into Th17 through interaction with activated dendritic cells (DCs) in the presence of IL-23; 2) Th17 producing IL-17A and IL-17F; and 3) keratinocyte stimulated by IL-17 ligands leading to aberrant differentiation and proliferation that promote the production of proinflammatory chemokines; and 4) angiogenic factors that stimulate further recruitment of inflammatory cells, setting up a positive feedback loop (Figure 1). ${ }^{32}$ In particular, it has been demonstrated that IL-23 produced by DCs and keratinocytes can induce IL-17A production by Th17 cells, Tc17 cells, $\gamma \delta \mathrm{T}$ cells, and innate cell subsets (mast cells and neutrophils). Furthermore, blood-derived ILC3 can produce IL-17A that activates fibroblasts to produce IL-6, IL-8, granulocyte colony-stimulating factor, and keratinocytes to produce IL-1 $\beta$, IL-6, IL-8, IL-17A, and TNF- $\alpha$, the latter being produced by several cell types including keratinocytes, DCs, and Th1 and Th17 cells. This cytokine cascade has an additive effect on keratinocytes leading to chemokines and AMP (including cathelicidin). Cathelicidin can bind to self-DNA- and self-RNA-forming complexes, which, respectively, trigger TLR9 and TLR7 activation in plasmacytoid DCs (pDCs). This is perceived as a danger signal, breaks immunological tolerance, and leads to the activation of pDCs and IFN- $\alpha$ secretion, and as a consequence, inflammasome and T-cell activation. IL-22 produced among others by TH17/Th22 cells - contributes to the onset of psoriasis by inducing keratinocyte proliferation and maturation..$^{27,30}$

\section{Ixekizumab and IL- I7A target therapies}

Based on these observations on the role of Th17 cells in psoriasis and PsA, three targeted agents, namely secukinumab, ixekizumab, and brodalumab, were developed. Secukinumab is a fully human IgG1 $\kappa$ monoclonal antibody approved by the Food and Drug Administration and the European Medicines Agency for the treatment of plaque psoriasis, PsA, and ankylosing spondylitis. ${ }^{33}$ Brodalumab is an IgG2 against IL-17 receptor, binding with high affinity to human IL-17RA, now undergoing phase III studies. ${ }^{34}$

Ixekizumab is an anti-IL-17A hinge-modified humanized immunoglobulin G subclass-4 (IgG4) antibody indicated for the treatment of moderate-to-severe plaque-type psoriasis. ${ }^{35,36}$ This molecule was assembled as a humanized IgG4 isotype to decrease the potential risk of immunogenicity caused by repeated administrations. ${ }^{36}$ Ixekizumab binds to human 


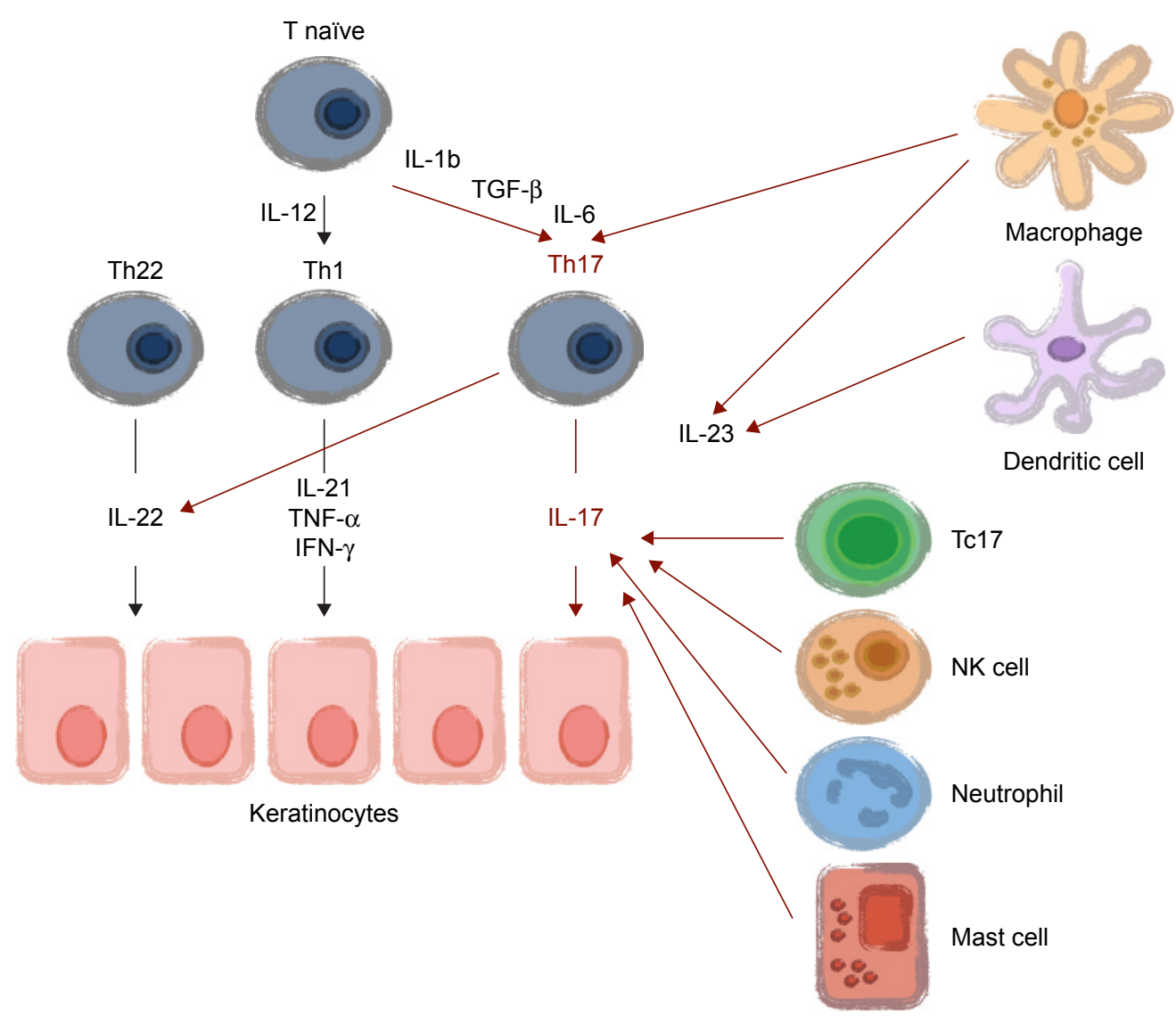

Figure I IL-17 in the pathogenesis of plaque-type psoriasis. Copyright @2014. Dove Medical Press. Modified from Lønnberg AS, Zachariae C, Skov L. Targeting of interleukin17 in the treatment of psoriasis. Clin Cosmet Investig Dermatol. 2014;7:25I-259.32

Abbreviations: IFN, interferon; IL, interleukin; TGF, tumor growth factor; TNF, tumor necrosis factor.

IL-17A at a conformational epitope, neutralizing the proinflammatory effect of IL-17A/F heterodimers in cell-based assays. ${ }^{37}$ It is administered subcutaneously; its recommended dose is $160 \mathrm{mg}$ at baseline, followed by $80 \mathrm{mg}$ in weeks 2, 4, $6,8,10$, and 12. After that, a maintenance dose of $80 \mathrm{mg}$ is administered every 4 weeks. The mean elimination half-life is 10.5 days. $^{25}$

\section{Clinical trials on plaque-type psoriasis Phase I}

The first phase I, randomized, double-blind, placebocontrolled, dose-escalation study on the safety and possible efficacy of ixekizumab in chronic moderate-to-severe plaque-type psoriasis was published in 2012 by Krueger et al. ${ }^{25}$ Ixekizumab was administered to 32 subjects with plaque psoriasis by subcutaneous injection across a range of doses from 5 to $150 \mathrm{mg}$ in weeks 0, 2, and 4. Eight subjects received placebo injections at the same time points. Patients were evaluated both clinically and histologically by skin biopsies taken before treatment and in weeks 2 and 6 . Histological evaluation demonstrated a marked reduction of both dermal infiltration of T cells and DCs and epidermal hyperplasia compared to baseline. Clinical efficacy, expressed as proportion of patients achieving a reduction in Psoriasis Area and Severity Index (PASI) score of at least 75\% from baseline (PASI75), has been summarized in Figure 2A. In week $6,25 \%, 71 \%$, and $100 \%$ of patients receiving 15,50 , and $150 \mathrm{mg}$, respectively, achieved PASI75. None of the patients receiving ixekizumab $5 \mathrm{mg}$ achieved the efficacy endpoint.

\section{Phase II}

Two phase II trials investigated the efficacy and safety of ixekizumab in the treatment of plaque-type psoriasis. In 2012, Leonardi et al, evaluated 142 patients affected by chronic moderate-to-severe plaque psoriasis in a double-blind, multicenter, randomized, dose-ranging study. The subjects were randomly assigned to receive subcutaneous injections of placebo or $10,25,75$, or $150 \mathrm{mg}$ of ixekizumab in weeks 0 , $2,4,8,12$, and $16 .^{38}$

The primary objectives were to assess whether ixekizumab was superior to placebo, by measuring the proportion of patients who achieved PASI75 in week 12, and to estimate 

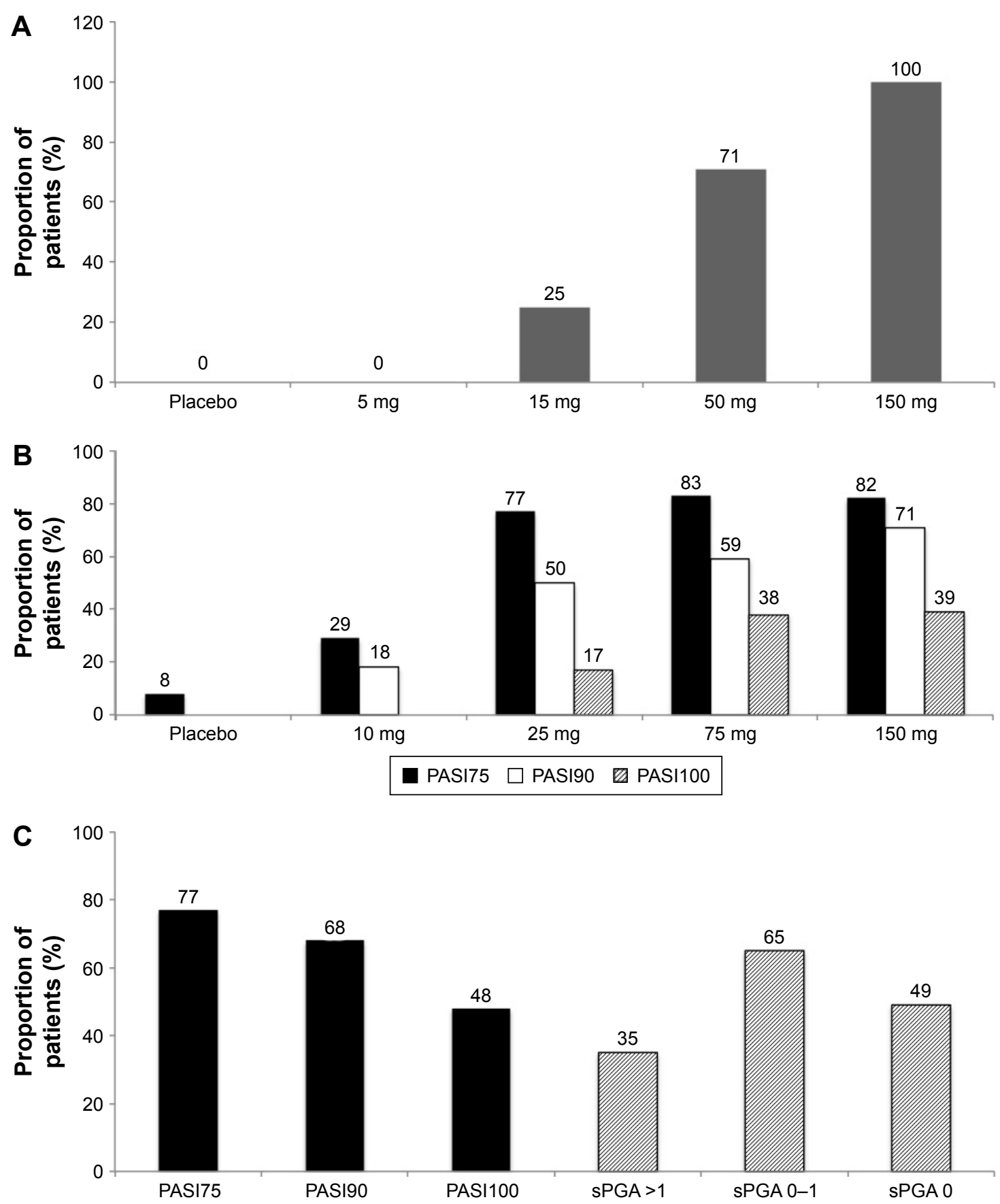

Figure 2 Efficacy of ixekizumab in phase I and phase II trials.

Notes: (A) Proportion of patients achieving PASI75 in phase I dose-escalation trial. In week 6, none of the subjects receiving placebo or 5 mg ixekizumab achieved PASI75, while 2/8, 5/7, and 8/8 subjects receiving 15, 50, or $150 \mathrm{mg}$ ixekizumab, respectively, accomplished the efficacy endpoint. ${ }^{25}$ (B) Proportion of patients achieving PASI75, 90 , and 100 in phase II study by Leonardi et al. ${ }^{38}$ After 12 weeks, PASI75 and PASI90 occurred in significantly more patients in the 25,75 , and I50 mg ixekizumab groups $(P<0.00$ I for each group vs placebo). Furthermore, a lot more patients in the 75 and $150 \mathrm{mg}$ groups achieved complete clearance (PASII00) ( $<<0.00 \mathrm{I}$ for each group vs placebo). ${ }^{38}$ (C) Proportion of patients achieving PASI75, 90, and 100 and sPGA values resulting in week 52 of the open-label extension of the phase II, randomized, placebocontrolled trial. ${ }^{39}$

Abbreviations: PASI, Psoriasis Area and Severity Index; sPGA, static Physician Global Assessment.

the percentage of reduction in the PASI score in each treatment group. The secondary objective included the proportion of patients with a reduced PASI score of at least $90 \%$ or $100 \%$ over the baseline value (PASI90 and PASI100, respectively), and the evaluation of the static physician's global assessment, joint pain visual analog scale, Nail Psoriasis Severity
Index (NAPSI), and Psoriasis Scalp Severity Index (PSSI). The resulting efficacy outcomes of the study have been summarized in Figure 2B.

Subsequently, a multicenter, open-label extension (OLE) of this phase II, randomized, placebo-controlled trial (RCT) was designed to evaluate the long-term ( 52 weeks) efficacy 
and safety of ixekizumab. ${ }^{39}$ Patients received ixekizumab $(10,25,75$, or $150 \mathrm{mg})$ or placebo subcutaneously in weeks 0 , $2,4,8,12$, and 16 of the RCT. In week 20 of the RCT, patients who did not reach PASI75 were eligible to enter the OLE and initiate monthly ixekizumab treatment. All other patients entered a treatment-free period from week 20 to week 32 and became eligible to enter the OLE at the study visit if PASI75 was lost or in week 32 if PASI75 or higher improvement was maintained throughout the treatment-free period. All patients were administered $120 \mathrm{mg}$ of ixekizumab subcutaneously every 4 weeks. Before entering the OLE, 69 of 120 (58\%) patients had achieved PASI75 in week 20 of the RCT and entered a treatment-free period from week 20 to 32 . Of these 69 patients, 45 (65\%) maintained a PASI75 response through the treatment-free period and entered the OLE in week 32. Twenty-four (35\%) patients entered the OLE before week 32 when their response was lower than PASI75. The remaining 51 patients were nonresponders in the RCT and entered the OLE in week 20. Efficacy was evaluated in week 52 through PASI and static Physician Global Assessment (sPGA). The observed results have been summarized in Figure 2C. Interestingly, the patients assigned to placebo during the RCT who did not achieve PASI75 were responsive during the OLE. In fact, a PASI75 response was observed in $95 \%$ (18 of 19), a PASI90 response was observed in 95\% (18 of 19), and a PASI100 response was observed in 63\% (12 of 19) of these patients.

\section{Phase III}

Three major multicenter, randomized, double-blind, placebo-controlled, parallel-group phase III studies have been published, namely UNCOVER-1, UNCOVER-2, and UNCOVER-3. ${ }^{40}$ Of these, UNCOVER-1 evaluated ixekizumab versus placebo, while UNCOVER-2 and UNCOVER-3 had an active comparator (etanercept). In these trials, investigators randomly assigned 1,296 patients to UNCOVER-1, 1,224 patients to UNCOVER-2, and 1,346 patients to UNCOVER-3.

UNCOVER-1 was a prospective, double-blind, multicenter trial investigating the efficacy of ixekizumab on inducing psoriasis clearance in 12 weeks on 1,296 patients. Subjects were randomly distributed in a 1:1:1 ratio to receive placebo or $80 \mathrm{mg}$ ixekizumab every 2 weeks (Q2W), $80 \mathrm{mg}$ ixekizumab every 4 weeks (Q4W) after an initial dose of $160 \mathrm{mg}$ at week 0 , respectively. Response to ixekizumab during the 12-week induction period has been summarized in Figure 3A.

In week 12, ixekizumab-responsive patients (sPGA 0 or 1 ) were randomly reassigned to receive placebo, $80 \mathrm{mg}$ of ixekizumab Q4W, or 80 mg of ixekizumab every 12 weeks up to week 60 .

UNCOVER-2 was a prospective, double-blind, multicenter 12-week study on 1,224 patients randomly distributed in a $2: 2: 2: 1$ ratio to receive subcutaneous placebo, etanercept (50 mg twice weekly), or one injection of $80 \mathrm{mg}$ ixekizumab every 2 weeks, or every 4 weeks after a $160 \mathrm{mg}$ starting dose. In addition to the co-primary endpoints of UNCOVER-1 (PASI75, 90, and 100, and sPGA 0 or 1 at week 12), this trial evaluated itch numeric rating scale and Dermatology Life Quality Index as secondary endpoints. The above-mentioned study design was repeated in the UNCOVER-3 trial for the first 12 weeks. Efficacy data in week 12 of UNCOVER-2 and UNCOVER-3 trials have been summarized in Figure 3B and C, respectively. A summary of ixekizumab 12-week efficacy observed in UNCOVER-1 and UNCOVER-2 trials is presented in Figure 3D.

In addition, UNCOVER-1 and UNCOVER-2 designs have an adjunctive randomized withdrawal period analysis from week 12 to week 60, while patients in the UNCOVER-3 trial received continuous treatment of ixekizumab from week 0 to 60 . The latter data have been summarized in Figure 3E.

A subanalysis of UNCOVER studies focused on scalp psoriasis demonstrated that in patients with moderate-tosevere psoriasis with baseline scalp involvement, PSSI 90 and 100 were achieved in week 12 in a higher percentage of patients treated with ixekizumab Q2W (81.7\% and 74.6\%) or ixekizumab Q4W (75.6\% and 68.9\%) compared with patients treated with placebo $(7.6 \%$ and $6.7 \% ; P<0.001$ each ixekizumab arm vs placebo) or etanercept (55.5\% and $48.1 \% ; P<0.001$ each ixekizumab arm vs etanercept). These outcomes were maintained in week 60 of the maintenance period of UNCOVER-1 and UNCOVER-2 trials. ${ }^{41}$

Similar results have been reported when a subanalysis focused on nail psoriasis was performed. ${ }^{42}$ In week 12 , ixekizumab Q2W or Q4W resulted in a greater improvement in nail psoriasis than placebo or etanercept, as measured by NAPSI reduction percentage (ixekizumab Q2W 39\% improvement, ixekizumab Q4W 40\%, etanercept 28\%, placebo $-4.7 \%$ ). In week 24 , a lot more patients receiving ixekizumab showed no signs of nail involvement $34 \%$ and 30\% in ixekizumab Q2W for 12 weeks then Q4W and ixekizumab Q4W, respectively). ${ }^{42,43}$

In addition to classical efficacy measures, a revision of UNCOVER trials data by Armstrong et al described the effect of ixekizumab on work productivity, measured by the Work Productivity and Activity Impairment-Psoriasis (WPAIPSO). ${ }^{44}$ The WPAI-PSO questionnaire was administered 

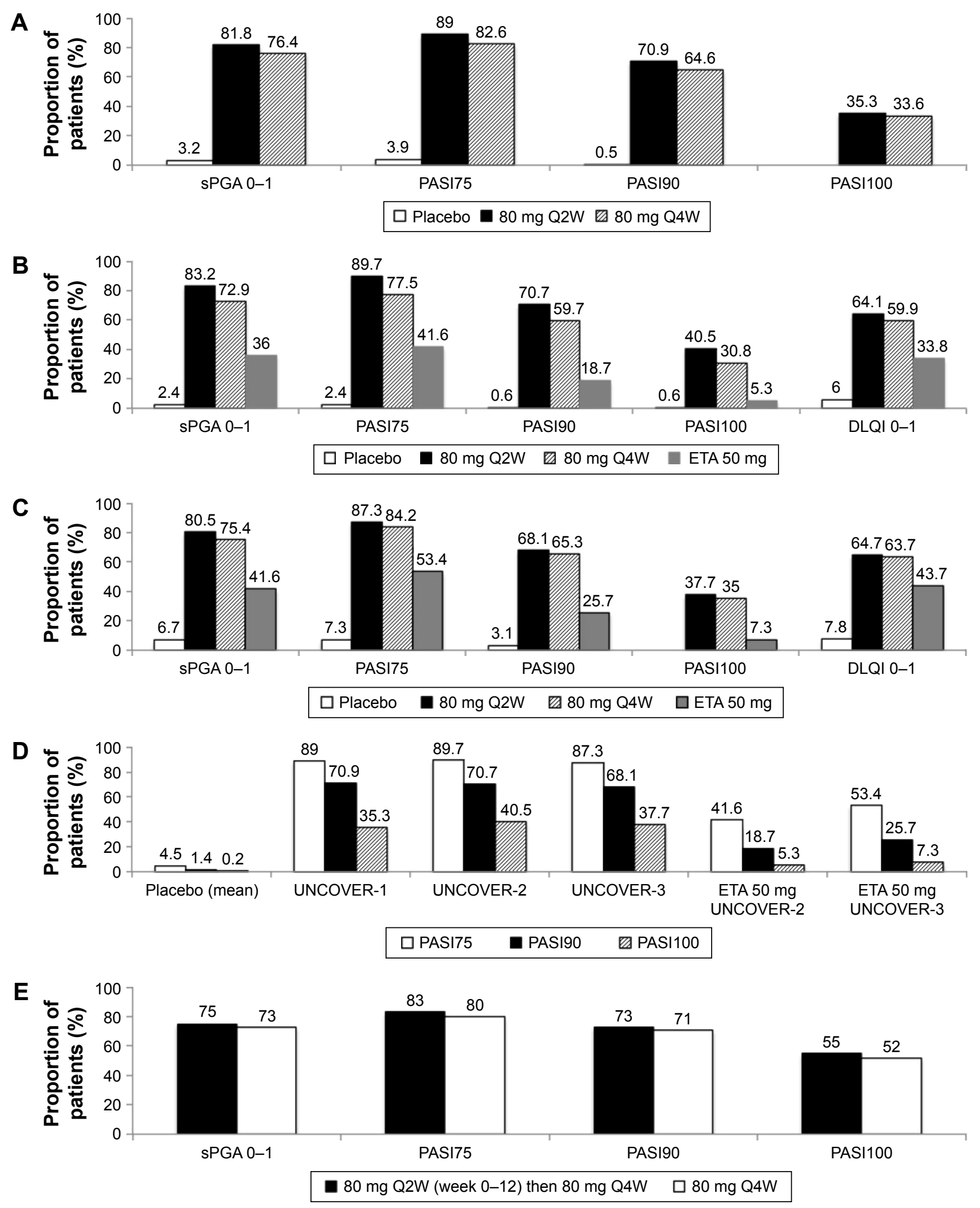

Figure 3 Efficacy of ixekizumab in phase III trials.

Notes: (A) Induction period in the UNCOVER-I trial: all comparisons of ixekizumab versus placebo were statistically significant $(P<0.00 \mathrm{I})$. (B) Results of the I2-week induction period of the UNCOVER-2.40 (C) Results of the 12-week induction period of the UNCOVER-3. (D) Summary of efficacy of ixekizumab 80 mg Q2W in inducing psoriasis clearance (expressed as proportion of patients achieving PASI75, 90, and I00 in week I2) compared to placebo (expressed as mean of the data resulting from the UNCOVER studies) or etanercept $50 \mathrm{mg}$ biweekly. (E) Maintenance of response to ixekizumab from baseline in week 60 (UNCOVER-3): maintenance of response to ixekizumab, at a dose of $80 \mathrm{mg}$, during the long-term extension period (week 12 of 60) among patients who had received ixekizumab Q2W during the induction period and patients who had received ixekizumab Q4W during the induction period.

Abbreviations: Q2W, every 2 weeks; Q4W, every 4 weeks; PASI, Psoriasis Area and Severity Index; sPGA, static Physician Global Assessment; ETA, etanercept. 
at baseline, at week 12 and at weeks 24, 36, 52, and 60 . The results of the questionnaire demonstrated that ixekizumab-treated patients had less impairment in work productivity than placebo-treated patients, and, within some items, less impairment than etanercept-treated patients.

Finally, UNCOVER-J was a phase III, multicenter, single-arm, open-label, long-term Japanese study. Eighty milligrams Q2W of ixekizumab was administered after a first dose of $160 \mathrm{mg}$. Subsequently, a maintenance dose of $80 \mathrm{mg}$ Q4W ixekizumab was administered. A total of 91 patients were enrolled, including 78 affected by moderate-to-severe plaque psoriasis, eight by erythrodermic psoriasis, and five by generalized pustular psoriasis. UNCOVER-J confirmed the efficacy in Japanese patients of ixekizumab (98.7\% of patients achieved PASI75) and suggest that ixekizumab could be clinically useful in patients with less common forms of psoriasis. However, the study population and the absence of controls represent a limit for this study. ${ }^{45}$

\section{Clinical trials on PsA}

Phase III data from the SPIRIT-P1 study showed that ixekizumab is safe and effective for the treatment of PsA, even though the included patients were naïve to biologics, including TNF- $\alpha$ inhibitors. ${ }^{46}$ The ongoing SPIRIT-P2 trial will provide data on patients previously exposed to biologics.

The SPIRIT-P1 study is a 3-year, phase III, randomized, double-blind, placebo-controlled and active-controlled trial, comparing two different regimens of ixekizumab with an active reference arm treated with adalimumab. The trial involved 417 patients randomized up to 24 weeks in four arms: 1) placebo ( $\mathrm{N}=106)$; 2) adalimumab $40 \mathrm{mg}$ biweekly ( $\mathrm{N}=101)$; 3) ixekizumab $80 \mathrm{mg}$ biweekly ( $\mathrm{N}=103)$; or 4) $80 \mathrm{mg}$ every 4 weeks $(\mathrm{N}=107)$ following $160 \mathrm{mg}$ initial dose. Among the 417 included subjects, 382 patients completed the 24-week study. Results demonstrated the significant efficacy of ixekizumab $80 \mathrm{mg}$ biweekly and ixekizumab $80 \mathrm{mg}$ every 4 weeks versus placebo in terms of ACR 20, ACR50, ACR70, and PASI 75/90/100 both after 12 and 24 weeks $(P<0.01)$. Furthermore, both ixekizumab regimens were effective versus placebo in reducing dactylitis after 12 and 24 weeks. Furthermore, the authors observed a significant improvement of disease activity and physical function, and the inhibition of PsA radiographic progression (modified Total Sharp Score [mTSS]).

\section{Safety}

Ixekizumab has been reported to be generally well tolerated without any organ toxicity. Severe adverse events were reported in $<2 \%$ of treated patients considering all the published studies. ${ }^{38-49}$ There were no deaths, and overall, an equal safety profile was observed between biologic-naïve and biologic-experienced patients. ${ }^{40,50}$ Severe adverse events were uncommon, with nasopharyngitis and upper-respiratory-tract infections as the most commonly reported. Nevertheless, they were equal among all treatment groups, indicating no added risk of these events attributable to ixekizumab. One major adverse cardiovascular event (MACE) was described in an ixekizumab-treated patient; similar adverse events were reported also in an etanercept-treated patient and in a placebotreated patient. ${ }^{40}$ These observations suggest that there is no specific risk for MACE determined by ixekizumab. One rectal cancer was reported in the long-term phase II trial. ${ }^{39}$ Furthermore, one patient experienced worsening of ulcerative colitis and one reported a new onset Crohn's disease during the first 12 weeks of treatment. ${ }^{48}$

There have been no suicides in psoriasis patients treated with ixekizumab; in contrast, two cases of suicide occurred in Brodalumab trials. ${ }^{40}$ Consistently with basic science data, Candida mucocutaneous infections have occurred in ixekizumab-treated patients, as observed with other antiIL-17A agents. ${ }^{51}$ Fungal infection seems to be related with higher doses of ixekizumab. They have been reported to be easily manageable with standard antimicrobial drugs; thus, none of the patients withdrew from the ixekizumab therapy. ${ }^{40}$ In summary, the safety profile of ixekizumab is encouraging and consistent with its mechanism of action. ${ }^{49}$

\section{Use in therapy and conclusion}

Since their introduction in clinical practice, biologics have raised the bar of therapeutic outcomes in psoriasis even though they are not effective in every case and a loss of response over time has been reported, particularly for monoclonal antibodies. ${ }^{51}$

Ixekizumab has proved to be significantly more effective than the soluble anti-TNF- $\alpha$ receptor in clearing plaque-type psoriasis with effects that are maintained in time..$^{40,49,51,52}$

The rapid onset of response determines a substantial gain in the patients' quality of life and work productivity. ${ }^{40,44}$ Furthermore, its efficacy has been confirmed also in difficultto-treat areas (ie, scalp and nails) $)^{44-46}$ and severe clinical variants of psoriasis (ie, erythrodemic psoriasis). ${ }^{41-43,45}$ Besides, ixekizumab has shown significant improvements in the activity of skin disease and in inhibiting radiographic progression in patients with concomitant iarthritis. ${ }^{46}$

The efficacy of ixekizumab is high both in patients with and without previous exposure to biologics. ${ }^{51}$ In particular, 
after switching to ixekizumab a substantial proportion of patients with moderate-to-severe psoriasis who did not respond to etanercept experienced rapid and durable improvement. ${ }^{48}$

It is difficult to estabilish an algorithm for sequential treatment in psoriasis patients since there are limited data on the efficacy of an anti-IL-17 agent in patients experiencing anti-TNF-alpha failure in real life..$^{52,53}$ Nevertheless, currently available phase I-III data indicate that ixekizumab is a promising drug, although long-term data on the efficacy and safety and real-world data are needed before ixekizumab and other IL-17-targeting agents can find their place in clinical practice. $^{49,52}$

Ixekizumab is an effective drug with a good safety profile and multiple target profile. It could be useful in patients with severe forms and multiple previous failures (second-/ third-line biologic) as well as a first-line biologic agent for the treatment of moderate-to-severe plaque-type psoriasis in patients unresponsive or contraindicated to conventional systemic agents.

\section{Disclosure}

A Giunta has served as a consultant and a speaker for Abbvie, Pfizer. MS Chimenti has served as a consultant and a speaker for Abbvie and Pfizer. M Esposito has served as a consultant and a speaker for Abbvie, Biogen, Novartis, Eli-Lilly, Pfizer. L Bianchi has served as a consultant, speaker, and a board member for Abbvie, Celgene, Janssen, Novartis, Pfizer, and UCB. A Ventura reports no conflicts of interest in this work.

\section{References}

1. Parisi R, Symmons DP, Griffiths CE, Ashcroft DM; Identification and Management of Psoriasis and Associated ComorbidiTy (IMPACT) project team. Global epidemiology of psoriasis: a systematic review of incidence and prevalence. J Invest Dermatol. 2013;133(2): 377-385.

2. Gordon KB, Strober BE. Understanding therapeutic pathways and comorbidities in psoriasis. Semin Cutan Med Surg. 2014;33(2 Suppl 2): S20-S23.

3. Davidovici BB, Sattar N, Prinz J, et al. Psoriasis and systemic inflammatory diseases: potential mechanistic links between skin disease and co-morbid conditions. J Invest Dermatol. 2010;130(7):1785-1796.

4. Gottlieb AB, Dann F. Comorbidities in patients with psoriasis. Am J Med. 2009;122(12):1150.e1-e9.

5. Gisondi P, Ferrazzi A, Girolomoni G. Metabolic comorbidities and psoriasis. Acta Dermatovenerol Croat. 2010;18(4):297-304.

6. Esposito M, Saraceno R, Giunta A, Maccarone M, Chimenti S. An Italian study on psoriasis and depression. Dermatology. 2006; 212(2):123-127.

7. Esposito M, Faleri S, Babino G, et al. From patients' needs to treatment outcomes in psoriasis: results from the pSORRIDI experience. J Int Med Res. 2016;44(1 Suppl):95-99.
8. Jacobs A, Rosumeck S, Nast A. Systematic review on the maintenance of response during systemic antipsoriatic therapy. Br J Dermatol. 2015; 173(4):910-921.

9. Park H, Li Z, Yang XO, et al. A distinct lineage of CD4 T cells regulates tissue inflammation by producing interleukin 17. Nat Immunol. 2005;6(11):1133-1141.

10. Cai Y, Fleming C, Yan J. Dermal $\gamma \delta$ T cells-a new player in the pathogenesis of psoriasis. Int Immunopharmacol. 2013;16(3):388-391.

11. Yao Z, Painter SL, Fanslow WC, et al. Human IL-17: a novel cytokine derived from T cells. J Immunol. 1995;155(12):5483-5486.

12. Gaffen SL, Jain R, Garg AV, Cua DJ. The IL-23-IL-17 immune axis: from mechanisms to therapeutic testing. Nat Rev Immunol. 2014; 14(9):585-600.

13. Gaffen SL. The role of interleukin-17 in the pathogenesis of rheumatoid arthritis. Curr Rheumatol Rep. 2009;11(5):365-370.

14. Kirkham BW, Lassere MN, Edmonds JP, et al. Synovial membrane cytokine expression is predictive of joint damage progression in rheumatoid arthritis: a two-year prospective study (the DAMAGE study cohort). Arthritis Rheum. 2006;54(4):1122-1131.

15. Katz Y, Nadiv O, Beer Y. Interleukin-17 enhances tumor necrosis factor alpha-induced synthesis of interleukin 1, 6 and 8 in skin and synovial fibroblasts: a possible role as a "fine-tuning cytokine" in inflammation process. Arthritis Rheum. 2001;44(9):2176-2184.

16. Chabaud M, Fossiez F, Taupin JL, Miossec P. Enhancing effect of IL-17 on IL-1-induced IL- 6 and leukemia inhibitory factor production by rheumatoid arthritis synoviocytes and its regulation by Th2 cytokines. J Immunol. 1998;161(1):409-414.

17. Jovanovic DV, Di Battista JA, Martel-Pelletier J, et al. IL-17 stimulates the production and expression of proinflammatory cytokines, IL- 1beta and TNF alpha, by human macrophages. J Immunol. 1998;160(7):3513-3521.

18. Chabaud M, Miossec P. The combination of tumor necrosis factor alpha blockade with interleukin-1 and interleukin-17 blockade is more effective for controlling synovial inflammation and bone resorption in an ex vivo model. Arthritis Rheum. 2001;44(6):1293-1303.

19. Ruddy MJ, Wong GC, Liu XK, et al. Functional cooperation between interleukin- 17 and tumor necrosis factor- $\alpha$ is mediated by CCAAT/ enhancer binding protein family members. J Biol Chem. 2004;279(4): 2559-2567.

20. Lubberts E. IL-17/Th17 targeting: on the road to prevent chronic destructive arthritis? Cytokine. 2008;41(2):84-91.

21. Benderdour M, Tardif G, Pelletier JP, et al. Interleukin 17 (IL-17) induces collagenase-3 production in human osteoclastic chondrocytes via AP-1 dependent activation: differential activation of AP-1 members by IL-17 and IL-1beta. J Rheum. 2002;29(21):1262-1272.

22. Koshy PJ, Henderson N, Logan C, Life PF, Cawston TE, Rowan AD. Interleukin 17 induces cartilage collagen breakdown: novel synergistic effects in combination with proinflammatory cytokines. Ann Rheum Dis. 2002;61(8):704-713.

23. Chiricozzi A. Pathogenic role of IL-17 in psoriasis and psoriatic arthritis. Actas Dermosifiliogr. 2014;105(Suppl 1):9-20.

24. Lowes MA, Russell CB, Martin DA, Towne JE, Krueger JG. The IL-23/ T17 pathogenic axis in psoriasis is amplified by keratinocyte responses. Trends Immunol. 2013;34(4):174-181.

25. Krueger JG, Fretzin S, Suárez-Fariñas M, et al. IL-17A is essential for cell activation and inflammatory gene circuits in subjects with psoriasis. J Allergy Clin Immunol. 2012;130(1):145.e9-154.e9.

26. Johansen C, Usher PA, Kjellerup RB, Lundsgaard D, Iversen L, Kragballe K. Characterization of the interleukin-17 isoforms and receptors in lesional psoriatic skin. Br J Dermatol. 2009;160(2):319-324.

27. Lowes MA, Kikuchi T, Fuentes-Duculan J, et al. Psoriasis vulgaris lesions contain discrete populations of Th1 and Th17 T cells. J Invest Dermatol. 2008;128(5):1207-1211.

28. Li J, Chen X, Liu Z, Yue Q, Liu H. Expression of Th17 cytokines in skin lesions of patients with psoriasis. J Huazhong Univ Sci Technolog Med Sci. 2007;27(3):330-332. 
29. Guttman-Yassky E, Lowes MA, Fuentes-Duculan J, et al. Low expression of the IL-23/Th17 pathway in atopic dermatitis compared to psoriasis. J Immunol. 2008;181(10):7420-7427.

30. Kagami S, Rizzo HL, Lee JJ, Koguchi Y, Blauvelt A. Circulating Th17, Th22, and Th1 cells are increased in psoriasis. J Invest Dermatol. 2010; 130(5):1373-1383.

31. Caproni M, Antiga E, Melani L, Volpi W, Del Bianco E, Fabbri P. Serum levels of IL-17 and IL-22 are reduced by etanercept, but not by acitretin, in patients with psoriasis: a randomized- controlled trial J Clin Immunol. 2009;29(2):210-214.

32. Lønnberg AS, Zachariae C, Skov L. Targeting of interleukin-17 in the treatment of psoriasis. Clin Cosmet Investig Dermatol. 2014;7: 251-259.

33. Rich P, Sigurgeirsson B, Thaci D, et al. Secukinumab induction and maintenance therapy in moderate-to-severe plaque psoriasis: a randomized, double-blind, placebo-controlled, phase II regimen-finding study. Br J Dermatol. 2013;168(2):402-411.

34. Russell CB, Rand H, Bigler J, et al. Gene expression profiles normalized in psoriatic skin by treatment with brodalumab, a human anti-IL-17 receptor monoclonal antibody. J Immunol. 2014;192(8): 3828-3836.

35. Genovese MC, Van den Bosch F, Roberson SA, et al. LY2439821, a humanized anti-interleukin-17 monoclonal antibody, in the treatment of patients with rheumatoid arthritis: a phase I randomized, doubleblind, placebo-controlled, proof-of-concept study. Arthritis Rheum. 2010;62(4):929-939.

36. Chan AC, Carter PJ. Therapeutic antibodies for autoimmunity and inflammation. Nat Rev Immunol. 2010;10(5):301-316.

37. Liu L, Lu J, Allan BW, et al. Generation and characterization of ixekizumab, a humanized monoclonal antibody that neutralizes interleukin 17A. J Inflamm Res. 2016;9:39-50.

38. Leonardi C, Matheson R, Zachariae C, et al. Anti-interleukin-17 monoclonal antibody ixekizumab in chronic plaque psoriasis. $N$ Engl J Med. 2012;366(13):1190-1199.

39. Gordon KB, Leonardi CL, Lebwohl M, et al. A 52-week, open-label study of the efficacy and safety of ixekizumab, an anti-interleukin-17A monoclonal antibody, in patients with chronic plaque psoriasis. $J \mathrm{Am}$ Acad Dermatol. 2014;71(6):1176-1182.

40. Gordon KB, Blauvelt A, Papp KA, et al; UNCOVER-1 Study Group; UNCOVER-2 Study Group; UNCOVER-3 Study Group. Phase 3 trials of Ixekizumab in moderate-to-severe plaque psoriasis. $N$ Engl J Med. 2016;375(4):345-356.

41. Reich K, Leonardi C, Lebwohl M, et al. Sustained response with ixekizumab treatment of moderate-to-severe psoriasis with scalp involvement: results from three phase 3 trials (UNCOVER-1, UNCOVER-2, UNCOVER-3). J Dermatolog Treat. 2016:1-6.

42. Dennehy EB, Zhang L, Amato D, Goldblum O, Rich P. Ixekizumab is effective in subjects with moderate to severe plaque psoriasis with significant nail involvement: results from UNCOVER 3. J Drugs Dermatol. 2016;15(8):958-961.
43. van de Kerkhof P, Guenther L, Gottlieb AB, et al. Ixekizumab treatment improves fingernail psoriasis in patients with moderate-to-severe psoriasis: results from the randomized, controlled and open-label phases of UNCOVER-3. J Eur Acad Dermatol Venereol. 2017;31(3):477-482.

44. Armstrong AW, Lynde CW, McBride SR, et al. Effect of Ixekizumab treatment on work productivity for patients with moderate-to-severe plaque psoriasis: analysis of results from 3 randomized phase 3 clinical trials. JAMA Dermatol. 2016;152(6):661-669.

45. Saeki H, Nakagawa H, Nakajo K, et al; Japanese Ixekizumab Study Group. Efficacy and safety of ixekizumab treatment for Japanese patients with moderate to severe plaque psoriasis, erythrodermic psoriasis and generalized pustular psoriasis: results from a 52-week, open-label, phase 3 study (UNCOVER-J). J Dermatol. 2017;44(4):355-362.

46. Mease PJ, van der Heijde D, Ritchlin CT, et al; SPIRIT-P1 Study Group. Ixekizumab, an interleukin-17A specific monoclonal antibody, for the treatment of biologic-naive patients with active psoriatic arthritis: results from the 24-week randomised, double-blind, placebo-controlled and active (adalimumab)-controlled period of the phase III trial SPIRIT-P1. Ann Rheum Dis. 2017;76(1):79-87.

47. Griffiths CE, Reich K, Lebwohl M, et al; UNCOVER-2 and UNCOVER-3 investigators. Comparison of ixekizumab with etanercept or placebo in moderate-to-severe psoriasis (UNCOVER-2 and UNCOVER-3): results from two phase 3 randomised trials. Lancet. 2015;386(9993):541-551

48. Blauvelt A, Papp KA, Griffiths CE, et al. Efficacy and safety of switching to Ixekizumab in Etanercept non-responders: a subanalysis from two phase III randomized clinical trials in moderate-to-severe plaque psoriasis (UNCOVER-2 and -3). Am J Clin Dermatol. 2017; 18(2):273-280.

49. Farahnik B, Beroukhim K, Zhu TH, et al. Ixekizumab for the treatment of psoriasis: a review of phase III trials. Dermatol Ther (Heidelb). 2016; 6(1):25-37.

50. Gottlieb AB, Lacour JP, Korman N, et al. Treatment outcomes with ixekizumab in patients with moderate-to-severe psoriasis who have or have not received prior biological therapies: an integrated analysis of two Phase III randomized studies. J Eur Acad Dermatol Venereol. Epub 2016 Oct 1.

51. Esposito M, Gisondi P, Cassano N, et al. Survival rate of antitumor necrosis factor-a treatments for psoriasis in routine dermatological practice: a multicenter observational study. Br J Dermatol. 2013;169(3): 666-672.

52. Dyring-Andersen B, Skov L, Zachariae C. Ixekizumab for treatment of psoriasis. Expert Rev Clin Immunol. 2015;11(4):435-442.

53. Elyoussfi S, Thomas BJ, Ciurtin C. Tailored treatment options for patients with psoriatic arthritis and psoriasis: review of established and new biologic and small molecule therapies. Rheumatol Int. 2016;36(5): 603-612.

\section{Publish your work in this journal}

Drug Design, Development and Therapy is an international, peerreviewed open-access journal that spans the spectrum of drug design and development through to clinical applications. Clinical outcomes, patient safety, and programs for the development and effective, safe, and sustained use of medicines are the features of the journal, which

\section{Dovepress}

has also been accepted for indexing on PubMed Central. The manuscript management system is completely online and includes a very quick and fair peer-review system, which is all easy to use. Visit http://www.dovepress.com/testimonials.php to read real quotes from published authors. 